\title{
Detecting early glaucomatous field defects with the size I stimulus and Statpac
}

Department of Ophthalmology, University of Cincinnat College of Medicine, Cincinnati, Ohio, USA A H Zalta J C Burchfield

Correspondence to: A H Zalta, MD, Mail Location 527, 231 Bethesda Avenue, Cincinnati, Ohio 45267-0527, USA.

Accepted for publication 14 December 1989

\begin{abstract}
The influence of stimulus size and normal database on the detection of visual field defects in automated static threshold perimetry (Humphrey Field Analyzer) was investigated in 82 eyes having a diagnosis of normal, glaucoma suspect, or early glaucoma. Using a mathematically derived 'normal' database, which assumes constantly decreasing threshold sensitivities with increasing eccentricity, the size I stimulus showed significantly greater sensitivity than the size III stimulus for detecting small, shallow scotomata in the central visual field. The use of Statpac, which contains an empirically derived, age-related normal database, increased the sensitivity significantly over that of the size III stimulus (with its mathematical model), and to a degree similar to that of the size I stimulus. The results obtained with the size I stimulus were reproducible and independent of the patient's age. This study suggests a potential role for the size I stimulus in evaluating eyes having or at risk of developing early glaucomatous field loss.
\end{abstract}

Automated threshold perimetry is a highly standardised, reproducible method of visual field testing which has replaced manual isoptre perimetry as the procedure of choice for detecting and following visual field defects in glaucoma. While the size I $\left(0.25 \mathrm{~mm}^{2}\right)$ stimulus is routinely used for plotting isoptres in manual Goldmann perimetry, the size III $\left(4.0 \mathrm{~mm}^{2}\right)$ stimulus has become the standard for automated threshold field testing. In automated perimetry the size III stimulus offers a larger, more uniform dynamic range and reduces the influence of optical blur as compared with the size I stimulus. ${ }^{12}$ Furthermore at maximum intensity the size I stimulus is not strong enough to quantify the depth of many defects in automated perimetry.

For all size stimuli on the Humphrey Field Analyzer mathematically derived 'normal' threshold values are used for comparison with the patient's measured threshold values in the determination of defect depth. In this mathematical model the perimeter calculates an expected 'normal' visual field contour for each stimulus size based on a slope equation which assumes a constant decrease in sensitivity for each degree of eccentricity in the visual field. Recently an age related normal database was incorporated into the Humphrey Field Analyzer statistical package, Statpac, ${ }^{5}$ for the size III stimulus. In this empirical model the perimeter uses age related threshold values derived from normal subjects as the expected normal visual field contour.
To our knowledge there have been no studies examining the potential uses of the smaller size I stimulus on the Humphrey Field Analyzer. We investigated the sensitivity of the size I stimulus compared with the size III stimulus for detecting early glaucomatous visual field defects in central static threshold perimetry. Both the mathematical model and Statpac's empirical model of normal were used with the size III stimulus for determination of defect depth. The sensitivities of these two models were compared with each other and with that of the size I stimulus. To evaluate further the utility of the smaller stimulus size we also studied the variability of results between initial (first) and subsequent (second) central threshold fields performed with the size I stimulus.

\section{Materials and methods}

Records of all patients seen in the glaucoma referral practice of one of the authors (AHZ) between September 1984 and July 1988 were reviewed. Those patients with eyes having a classification of normal, glaucoma suspect, or early glaucoma and who had undergone central static threshold visual field testing with both size I and size III stimuli were evaluated retrospectively for the presence and density of visual field defects. A defect was defined as a subthreshold locus in the visual field, at least $0.6 \mathrm{log}$ unit in depth, as determined by comparing actual and expected (normal) threshold values at each point in the visual field.

An eye classified as glaucoma suspect had either intraocular pressures greater than 21 $\mathrm{mmHg}$ or suspicious asymmetric cupping of the optic nerves. Early glaucoma was defined by intraocular pressure greater than $21 \mathrm{mmHg}$ and either early visual field loss on at least two occasions or early optic nerve damage as confirmed by stereo disc photographs. The visual field defect in early glaucoma consisted of relative nerve fibre bundle defects at least $0.6 \mathrm{log}$ unit in depth involving only a single hemifield and an absence of other optic nerve or chorioretinal disease that might account for the field loss. In the absence of early visual field loss an eye may qualify for the designation of early glaucoma by having either optic nerve cupping at least $15 \%$ greater than the contralateral optic nerve or documented progression of cupping by stereo disc photographs.

All central static threshold visual fields were performed with the central 30-2 program on the Humphrey Field Analyzer Model 620 according to the manufacturer's recommendations. ${ }^{4} \mathrm{~A}$ 'full' threshold strategy was used for all initial examination and a 'full from prior' threshold strategy was used for all subsequent examina- 
Figure 1: Defect depth printouts for central static threshold visual fields in an eye with early glaucoma for the size I stimulus using a mathematically derived normal database (left), the size III stimulus using Statpac's empirically derived normal database (middle), and the size III stimulus using a mathematically derived normal database (right) Boxed loci represent $\geqslant 6 d B$ Boxed loci represent $\geqslant 6$
deviations from normal.
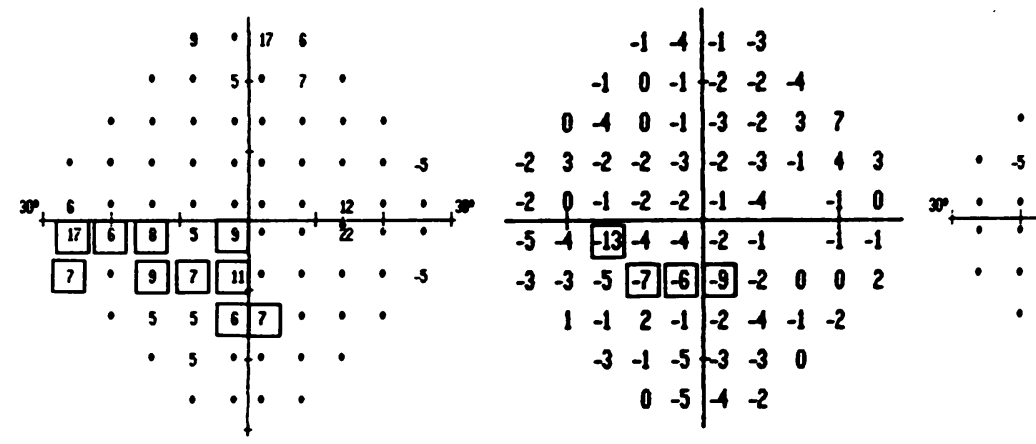

tions. The Humphrey Field Analyzer was updated with all software revisions as they became available and with Statpac. ${ }^{5}$

Exclusion criteria were established to eliminate the influences of learning effect, change in treatment or diagnosis, poor testing reliability, and artefact. We excluded eyes and visual fields with the following conditions: (1) any field which is a patient's first experience with automated threshold testing; (2) pupils that could not be dilated to at least $3 \mathrm{~mm}$ in diameter; (3) media opacity reducing visual acuity to less than 20/40; (4) clinically unstable early glaucoma requiring additional ocular hypotensive therapy; (5) consistent low reliability on visual field testing as defined by fixation losses greater than $33 \%$, false positive errors greater than $25 \%$, or false negative errors greater than $25 \%$; and (6) unreproducible edge artefact due to a trial lens rim or an intermittently droopy eyelid or prominent eyelashes. In an effort to minimise artefact the superior and temporal four threshold points at $27^{\circ}$ eccentricity were excluded from analysis.

For each eye fulfilling the above criteria three different defect depth printouts were obtained (Fig 1). Numeric defect depth printouts which used mathematically derived 'normal' threshold values were obtained for both stimulus size $I$ and size III fields. In this mathematical model the perimeter uses a slope value of $0.40 \mathrm{~dB}$ per degree of eccentricity for the size I stimulus and a value of $0.31 \mathrm{~dB}$ per degree of eccentricity for the size III stimulus. ${ }^{4}$ Moreover the numeric pattern deviation display on the single field analysis printout, which uses Statpac's empirically derived normal threshold values, was obtained for each stimulus size III field. As seen in Figure
1 , defect depth is reported as a positive value for the mathematical model (right and left) and as a negative value for the empirical model (centre).

Defects on these three printouts were classified by the number and depth of contiguous subnormal threshold points. The defect criteria for size were $1,2,3$, and 4 adjacent loci and for depth were 6,7 , and $8 \mathrm{~dB}$. A field was said to fulfil a given criterion if any defect in the field was of equal or greater size and depth than that criterion.

Variability of results obtained with the size I stimulus was examined in a subgroup of subjects who had undergone more than one central static threshold field with the size I stimulus. Numeric defect depth printouts from the first and second tests on each eye were compared by the aforementioned defect criteria (Fig 2).

A statistical analysis was performed by the Wilcoxon matched pairs signed-rank test. ${ }^{6}$ The actual calculations were made by the App-Stat software package for the Apple IIe computer. ${ }^{7}$ Although only two separate visual field examinations were performed for purposes of size I and size III stimulus comparisons, three evaluative methods were used, and hence three separate pairwise comparisons were made. Therefore, in an attempt to limit the probability of type I error statistical significance is claimed at the two-tailed 0.02 rather than 0.05 significance level. Probability values between 0.02 and 0.05 were considered of only marginal significance. The standard two-tailed 0.05 significance level was used for the single pairwise comparison between results from initial and repeat stimulus size I fields.

To determine if age was a confounding variable in the use of the size I stimulus, subjects in
Figure 2: Defect depth printouts for first (left) and second (right) stimulus size I central static threshold visual fields performed three months apart in an eye suspected of having glaucoma. Boxed loci represent $\geqslant 6 d B$ deviations from normal.

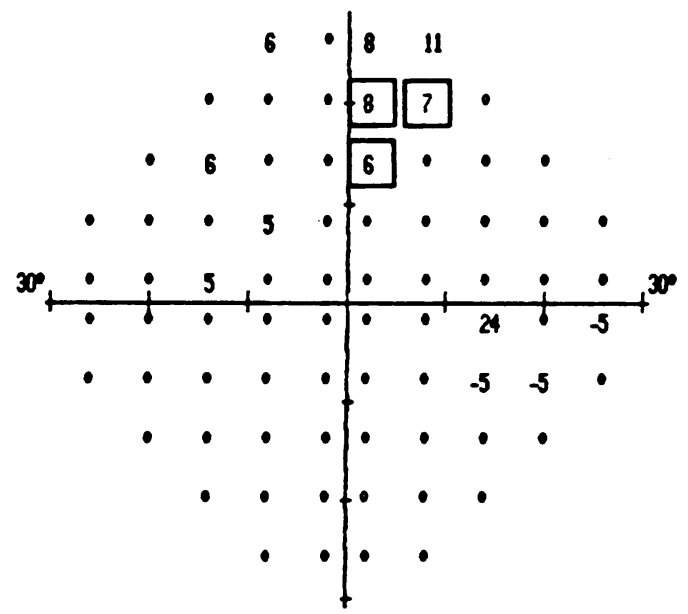


the glaucoma suspect and early glaucoma populations were divided into two subgroups: those aged 55 and under and those over age 55. A separate analysis with the Mann-Whitney $U$ test $^{6}$ was then performed comparing results from the initial stimulus size I fields for these two age groups.

\section{Results}

A total of 82 eyes fulfilled the study criteria and had central threshold fields performed with both the size I and size III stimuli. Of these eyes, 10 were normal, 46 were glaucoma suspect, and 26 had early glaucoma. The mean age (with SD) of all eyes was $50.1(14.9)$ years, with mean ages of $35 \cdot 5(9 \cdot 7)$ years for the normal group, 50.1 (16.2) years for the glaucoma suspect group, and $55 \cdot 7$ (9.8) years for the early glaucoma group. The average length of time (with SD) between performance of stimulus size I and size III fields was 21 (30) weeks (range 0-116 weeks).

Results of overall comparisons of the three evaluative methods are shown in Figure 3 and Table I. For defect depth determination using only the mathematical model the size I stimulus showed significantly greater sensitivity than the size III stimulus for nearly all defect criteria. In the comparison of the two models of defect depth determination with the size III stimulus, Statpac's empirical model showed significantly greater sensitivity than the mathematical model at most defect criteria. With a single exception the sensitivity of the size III stimulus using Statpac's empirical model was not significantly different from that of the size I stimulus for any defect criteria.

In the subgroup of normal eyes there were no significant differences between any of the three evaluative methods for any defect criteria with a single exception. For defects consisting of a single $6 \mathrm{~dB}$ locus, the size III stimulus with the empirical model was more sensitive than the size III stimulus with the mathematical model.

Of the 82 eyes 42 had second central threshold fields performed with the size I stimulus. Diagnostically, four eyes were normal, 24 were glaucoma suspect, and 14 had early glaucoma. The average length of time (with SD) between initial and subsequent size I stimulus fields was 22 (27) weeks (range 0-109 weeks). Comparison of results from initial and repeat stimulus size I fields revealed no significant differences $(p>0.05)$ in sensitivity between the two tests for all criteria of defect size and depth.

In the glaucoma suspect group there were 20 eyes of patients over age 55 and 26 eyes of patients aged 55 or younger. In the early glaucoma group there were 13 eyes of patients over age 55 and 13 eyes of patients aged 55 or younger. For fields performed with the size I stimulus a comparison of results between the two age groups revealed no significant differences for all defect criteria for both diagnostic groups.

\section{Discussion}

The psychophysical effects of the different-sized stimuli used in manual Goldmann perimetry and automated threshold perimetry have been pre-
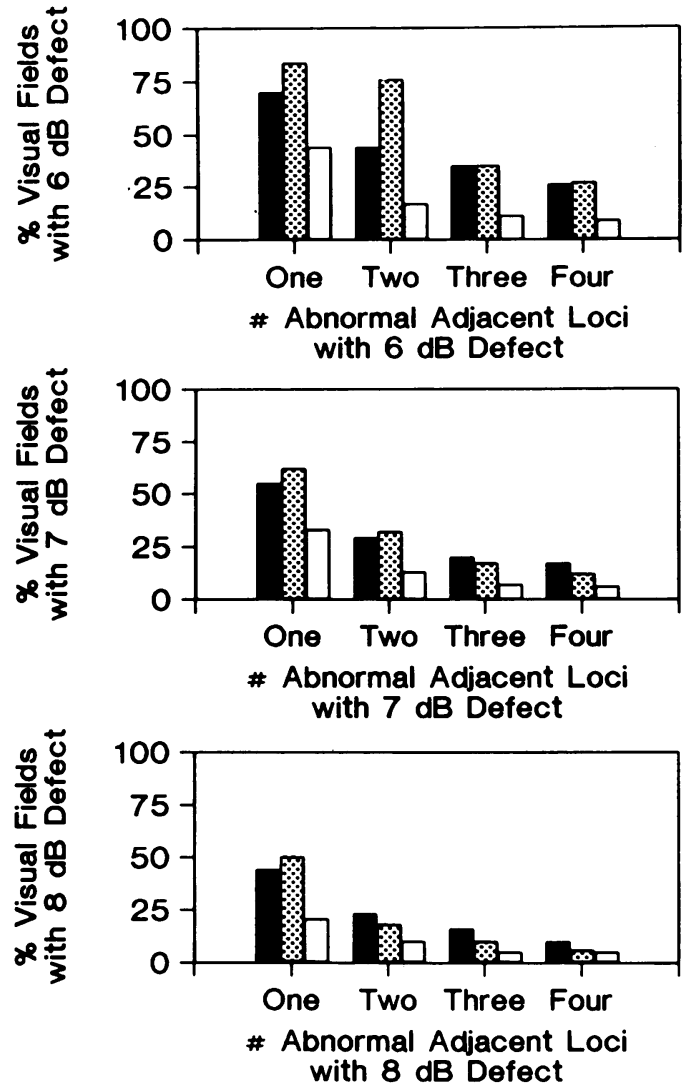

Size I Stimulus - math

Size III Stimulus - empiric (Statpac)

Size III Stimulus - math

Figure 3: Percentage of visual fields fulfilling defect criteria for the size I stimulus using a mathematically derived normal database (size I stimulus-math), the size III stimulus using Statpac's empirically derived normal database (size III stimulus-empirical), and the size III stimulus using a mathematically derived normal database (size III stimulus-math).

viously studied. ${ }^{289}$ However, little research has been done on the clinical applications of nonstandard stimuli in automated perimetry. Gramer et $a l^{10}$ compared the stimulus size I and size III in 12 eyes and the stimulus size III and size $\mathrm{V}$ in 18 eyes with glaucoma. They found that when 'using large test targets, small scotomata can be overlooked and identified field defects appear smaller and less deep'. More recently Wilensky $e t a l^{11}$ demonstrated the potential usefulness of the larger size $\mathrm{V}\left(64 \mathrm{~mm}^{2}\right)$ stimulus in testing eyes with advanced glaucoma. To the best of our knowledge there have been no studies examining the clinical utility or sensitivity of the size I stimulus on the Humphrey Field Analyzer.

When a mathematically derived 'normal' database is used in the determination of defect depth, we discovered that the size I stimulus shows significantly greater sensitivity in detecting, small, shallow scotomata than the size III stimulus. This finding may be explained by the concept of spatial summation. It is well known that because of spatial summation a larger stimulus is more easily seen than a smaller stimulus of the same luminance. ${ }^{23}$ The increased sensitivity of the size I stimulus may be due to stimulation of a smaller retinal receptive field with a consequent reduction in spatial summa- 
TABLE I Overall comparisons of sensitivity of size I and size III stimuli using mathematically and empirically derived normal databases ${ }^{\star}$

\begin{tabular}{|c|c|c|c|}
\hline \multirow{2}{*}{$\begin{array}{l}\text { Defect criteria, } \\
\text { size }(\text { adjacent } \\
\text { loci } \times \text { depth }(d B)\end{array}$} & \multicolumn{3}{|c|}{ Probability values } \\
\hline & $\begin{array}{l}\text { I-math } \mathrm{v} \\
\text { III-empiric }\end{array}$ & $\begin{array}{l}I-\text { math } \mathrm{v} \\
I I I-\text { math }\end{array}$ & $\begin{array}{l}\text { III-empiric } \\
\text { v III-math }\end{array}$ \\
\hline $\begin{array}{l}1 \times 6 \\
2 \times 6 \\
3 \times 6 \\
4 \times 6\end{array}$ & $\begin{array}{l}0.02 \\
\text { NS } \\
\text { NS } \\
\text { NS }\end{array}$ & $\begin{array}{l}0.002 \\
0.0007 \\
0.0009 \\
0.009\end{array}$ & $\begin{array}{l}0.0001 \\
0.0008 \\
0.0002 \\
0.001\end{array}$ \\
\hline $\begin{array}{l}1 \times 7 \\
2 \times 7 \\
3 \times 7 \\
4 \times 7\end{array}$ & $\begin{array}{l}\text { NS } \\
\text { NS } \\
\text { NS } \\
\text { NS }\end{array}$ & $\begin{array}{l}0.007 \\
0.01 \\
0.02 \\
0.04\end{array}$ & $\begin{array}{l}0.0001 \\
0.001 \\
0.01 \\
0.03\end{array}$ \\
\hline $\begin{array}{l}1 \times 8 \\
2 \times 8 \\
3 \times 8 \\
4 \times 8\end{array}$ & $\begin{array}{l}\text { NS } \\
\text { NS } \\
\text { NS } \\
\text { NS }\end{array}$ & $\begin{array}{l}0.002 \\
0.01 \\
0.01 \\
\text { NS }\end{array}$ & $\begin{array}{l}0.0001 \\
0.04 \\
\text { NS } \\
\text { NS }\end{array}$ \\
\hline
\end{tabular}

*I-math indicates size I stimulus using mathematically derived normal database; III-empiric indicates size III stimulus using Statpac's empirically derived normal database; and III-math indicates size III stimulus using mathematically derived normal database. NS, not significant.

tion, as compared with the size III stimulus. The principle is the converse of that of using the larger size $\mathrm{V}$ stimulus in advanced glaucoma to map threshold values in areas of the visual field unresponsive to the standard size III stimulus. In this case the smaller size I stimulus detects subthreshold responses in areas of the visual field mapped as normal by the less sensitive size III stimulus.

The size III stimulus, however, does have certain diagnostic advantages. It offers a larger and more uniform dynamic range than the size I stimulus, particularly in the less sensitive peripheral field, where spatial summation is inherently greater. ${ }^{28}$ Also, by extending the dynamic range, the size III stimulus 'makes it possible to record the sensitivity distribution in areas of maximal reduction of sensitivity'. ${ }^{2}$ In screening for early glaucomatous visual field loss, however, one is not examining the peripheral field nor attempting to quantify severe abnormalities in the central field. Rather, one is attempting to detect shallow paracentral scotomas. Hence a more desirable psychophysical property in such an application is maximal sensitivity in the central field. In threshold field testing of glaucoma suspects or eyes with early glaucoma, increased dynamic range with the size III stimulus is not of real practical value. In fact the smaller size I stimulus, which excites a lesser degree of spatial summation, may be a more logical stimulus choice. This reasoning is supported by the greater sensitivity of the size I stimulus than the larger size III stimulus seen in this study.

What is the clinical significance of the increased sensitivity of the size I stimulus over the more commonly used size III stimulus when a mathematically derived 'normal' database is used in determination of defect depth? Does it merely create false positive defects, or does it provide data which can be used in following a patient's clinical course or in making a decision to initiate treatment? In the subgroup of normal eyes there were no significant differences between results from the size I and III stimuli for any of the defect criteria. This supports our belief that the sensitivity of the size I stimulus is real and not simply derived from the creation of false positive defects. Furthermore, repeat field testing with the size I stimulus showed that these small, shallow scotomas were reproducible and independent of the patient's age. We believe that the increased sensitivity of the size I stimulus provides clinically useful information for evaluating and following up patients at risk of developing, or who already have, early glaucomatous visual field loss.

In addition, the sensitivity of the size III stimulus using an empirical model closely matched that of the size I stimulus using a mathematical model. To explain this finding we must assume that the increase in sensitivity resulting from use of the smaller size I stimulus is similar in degree to that of using an empirically derived database with the size III stimulus. Heijl et $a l^{12}$ have shown that there is significant location dependent variability of threshold values across the central visual field for the standard size III stimulus on a Humphrey Field Analyzer. This normal variability is not accounted for by the slope equation in the mathematical model but is inherently accounted for by the age related empiric database. Perhaps the use of an empirically derived normal database with the size I stimulus might further increase the sensitivity of threshold perimetry in glaucoma suspects and patients with early glaucoma by combining the benefits of both a smaller stimulus size and a more accurate approximation of expected threshold values.

When a smaller target size is used in perimetry, there is a greater influence of light diffusion caused by opacities of the media. ${ }^{2}$ In this study we excluded all eyes having media opacity which reduced visual acuity to less than $20 / 40$. In the presence of significant media opacity there may be a high level of background noise on field testing with the size I stimulus. Larger stimulus sizes may provide more usefu information when the transparency of the dioptic media has suffered. ${ }^{2}$

The current set of standard parameters used in automated threshold perimetry provide flexibility and sensitivity in a broad range of applications. While the size III stimulus may be appropriate for routine perimetry, our results suggest a role for the size I stimulus in screening for early glaucomatous visual field defects. As this study and Wilensky et al" have shown, certain specialised applications of threshold perimetry might be better served by nonstandard parameters such as the size I or size $V$ stimulus. As more of these specialised applications are discovered, and with the development of the corresponding perimetry software, it may be possible, through the selection of different stimulus sizes, to tailor visual field tests specifically to a patient's clinical status in order to obtain the most useful information for diagnosis and treatment.

This study was supported in part by an unrestricted grant from Research to Prevent Blindness, Inc, New York.

The authors have no direct or indirect commercial or proprietary interest in the Humphrey Field Analyzer or any other product mentioned in this report.

1 Fankhauser $F$. The development of computerized perimetry. In: Whalen WR, Spaeth GL, eds. Computerized visual fields. What they are and how to use them. Thorofare, NJ: Slack, 1985: 14. 
2 Fankhauser F. Problems related to the design of automated perimeters. Doc Ophthalmol 1979; 47: 89-138.

3 Anderson DR. Perimetry with and without automation. 2nd ed. St Louis: Mosby, 1987: 43.

4 Owner's Manual, Humphrey Field Analyzer. San Leandro, CA: Allergan Humphrey, 1983: sect. 3.3, 4, 5.1, 6, 9.1,
9.

5 . 9.2 . Heijl A, Lindgren G, Olsson J. A Package for the statistical
analysis of visual fields. Doc Ophthalmol Proc Ser 1987; 49:
153-68. $153-68$

6 Snedecor GW, Cochran WG. Statistical methods. 7th ed. Ames: Iowa State University Press, 1980: ch 8

7 App-STAT Statistical Package for Apple II Computers. Tulsa:

8 Sloan LL. Area and luminance of test object as variables in examination of the visual field by projection perimetry. Vision Res 1961; 18: 121-38.

9 Johnson CA, Keltner JL, Balestrery F. Effects of target size and eccentricity on visual detection and resolution. Vision

10 Gramer E, Kontic D, Krieglstein GK. Die computerperimetrische Darstellung glaukomatoser Gesichtsfelddefekte in Abhangigkeit von der Stimulusgrosse. Ophthalmologica 1981; 183: 162-7.

11 Wilensky JT, Mermelstein JR, Siegel HG. The use of different-sized stimuli in automated perimetry. $A m \mathcal{F}$ Ophthalmol 1986; 101: 710-3.

12 Heijl A, Lindgren G, Olsson J. Normal variability of static perimetric threshold values across the central visual field. Arch Ophthalmol 1987; 105: 1544-9. 